University of Wollongong

Research Online

2019

Utilisation patterns of privately funded mental health services in Australia

Joanna Khoo

University of Wollongong, jk645@uowmail.edu.au

Helen M. Hasan

University of Wollongong, hasan@uow.edu.au

Kathy Eagar

University of Wollongong, keagar@uow.edu.au

Follow this and additional works at: https://ro.uow.edu.au/ahsri

Research Online is the open access institutional repository for the University of Wollongong. For further information contact the UOW Library: research-pubs@uow.edu.au 


\title{
Utilisation patterns of privately funded mental health services in Australia
}

\begin{abstract}
Purpose: The purpose of this paper is twofold: first, to present patient-level utilisation patterns of hospitalbased mental health services funded by private health insurers; and second, to examine the implications of the findings for planning and delivering private mental health services in Australia. Design/ methodology/approach: Analysing private health insurance claims data, this study compares differences in demographic and hospital utilisation characteristics of 3,209 patients from 13 private health insurance funds with claims for mental health-related hospitalisations and 233,701 patients with claims for other types of hospitalisations for the period May 2014 to April 2016. Average number of overnight admissions, length of stay and per patient insurer costs are presented for each group, along with overnight admissions vs same-day visits and repeat services within a 28-day period following hospitalisation. Challenges in analysing and interpreting insurance claims data to better understand private mental health service utilisation are discussed. Findings: Patients with claims for mental health-related hospitalisations are more likely to be female (62.0 per cent compared to 55.8 per cent), and are significantly younger than patients with claims for other types of hospitalisations (32.6 per cent of patients aged 55 years and over compared to 57.1 per cent). Patients with claims for mental health-related hospitalisations have significantly higher levels of service utilisation than the group with claims for other types of hospitalisations with a mean length of stay per overnight admission of 15.0 days $(S D=14.1)$, a mean of 1.3 overnight admissions annually $(S D=1.2)$ and mean hospital costs paid by the insurer of $\$ 13,192$ per patient $(S D=13,457)$ compared to 4.6 days $(S D=7.3), 0.8$ admissions $(S D=0.6)$ and $\$ 2,065$ per patient $(S D=4,346)$, respectively, for patients with claims for other types of hospitalisations. More than half of patients with claims for mental health-related hospitalisations only claim for overnight admissions. However, the findings are difficult to interpret due to the limited information collected in insurance claims data. Practical implications: This study shows the challenges of understanding utilisation patterns with one data source. Analysing insurance claims reveals information on mental health-related hospitalisations but information on community-based care is lacking due to the regulated role of the private health insurance sector in Australia. For mental health conditions, and other chronic health conditions, multiple data sources need to be integrated to build a comprehensive picture of health service use as care tends to be provided in multiple settings by different medical and allied health professionals. Originality/value: This study contributes in two areas: patient-level trends in hospital-based mental health service utilisation claimed on private health insurance in Australia have not been previously reported. Additionally, as the amount of data routinely collected in health care settings increases, the study findings demonstrate that it is important to assess the quality of these data sources for understanding service utilisation.
\end{abstract}

\section{Publication Details}

J. Khoo, H. Hasan \& K. Eagar, "Utilisation patterns of privately funded mental health services in Australia", Journal of Health Organization and Management 331 (2019) 5-17. 


\title{
Utilisation patterns of privately funded mental health services in Australia
}

\author{
Joanna Khoo*1,2, Helen Hasan ${ }^{1}$, Kathy Eagar ${ }^{1}$
}

\author{
${ }^{1}$ Australian Health Services Research Institute, University of Wollongong, Australia \\ ${ }^{2}$ Health Market Quality Program, Capital Markets Cooperative Research Centre,
} Sydney, Australia

*Corresponding author: Email: jk645@uowmail.edu.au

\begin{abstract}
Purpose: To present patient-level utilisation patterns of hospital-based mental health services funded by private health insurers, and examine the implications of the findings for planning and delivering private mental health services in Australia.

Design/methodology/approach: Analysing private health insurance claims data, this study compares differences in demographic and hospital utilisation characteristics of 3,209 patients from 13 private health insurance funds with claims for mental healthrelated hospitalisations and 233,701 patients with claims for other types of hospitalisations for the period May 2014 to April 2016. Average number of overnight admissions, length of stay, and per patient insurer costs are presented for each group, along with overnight admissions versus same-day visits and repeat services within a 28-day period following hospitalisation. Challenges in analysing and interpreting insurance claims data to better understand private mental health service utilisation are discussed.
\end{abstract}

Findings: Patients with claims for mental health-related hospitalisations are more likely to be female (62.0\% compared to $55.8 \%$ ), and are significantly younger than patients with claims for other types of hospitalisations (32.6\% of patients aged 55 years and over compared to 57.1\%). Patients with claims for mental health-related hospitalisations have significantly higher levels of service utilisation than the group with claims for other types of hospitalisations with a mean length of stay per overnight admission of 15.0 days ( $\mathrm{SD}=14.1$ ), a mean of 1.3 overnight admissions annually ( $\mathrm{SD}=1.2$ ) and mean hospital costs paid by the insurer of $\$ 13,192$ per patient $(\mathrm{SD}=13,457)$ compared to 4.6 days $(\mathrm{SD}=7.3), 0.8$ admissions $(\mathrm{SD}=0.6)$ and $\$ 2,065$ per patient $(S D=4,346)$ respectively for patients with claims for other types of hospitalisations. More than half of patients with claims for mental health-related 
hospitalisations only claim for overnight admissions. However, the findings are difficult to interpret due to the limited information collected in insurance claims data.

Practical implications: This study shows the challenges of understanding utilisation patterns with one data source. Analysing insurance claims reveals information on mental health-related hospitalisations but information on community-based care is lacking due to the regulated role of the private health insurance sector in Australia. For mental health conditions, and other chronic health conditions, multiple data sources need to be integrated to build a comprehensive picture of health service use as care tends to be provided in multiple settings by different medical and allied health professionals.

Originality/value: This study contributes in two areas: Patient-level trends in hospital-based mental health service utilisation claimed on private health insurance in Australia have not been previously reported. Additionally, as the amount of data routinely collected in health care settings increases, the study findings demonstrate that it is important to assess the quality of these data sources for understanding service utilisation. 


\section{Introduction}

Some people living with a mental health condition require care over a long period of time provided by health professionals in both hospital and community settings, due to the chronic and persistent nature of the condition. The term "mental health condition" refers to a diverse spectrum of disorders including anxiety, depression, substance use disorders and schizophrenia. These conditions vary in their severity, duration and prevalence in the general population. Australia has a mixed public-private health insurance and service delivery system in which mental health care is funded by both government sources and private sources through private health insurance and patient contributions. Internationally, mental health care may also have mixed funding sources or limited insurance coverage (Sharfstein 1995) and there is often a clear distinction, and resulting lack of integration, between psychiatric and other forms of medical care (Vladu et al., 2016).

Although Australia dedicates a greater level of resources to mental health care than many other countries (Saxena et al., 2007), national figures report only 65\% of people with a mental health disorder classified as severe accessed health services for the issue in the previous 12 months (Australian Government Department of Health, 2013). A range of factors influences the utilisation of health services. These factors have been organised into contextual and individual factors in the Behavioural Model of Health Service Use (Andersen, 1995; Andersen et al., 2014). Factors may be predisposing characteristics or health beliefs that influence access to care or they may be factors such as the policies, financing and organisation of the health system that enable or impede access to care (Aday and Andersen, 1974; Andersen et al., 2014). The influence of contextual, enabling factors identified in the Model such as health care organisation and financing, frames the analysis and measurement of utilisation 
patterns in this study. The study analyses insurance claims data to examine the type, the organisation and the frequency of mental health services obtained by patients with private health insurance.

In Australia, individuals can access hospital-based mental health services using private health insurance ranging from individual and group-based psychological therapies to intensive, inpatient treatment that can last for several weeks. Medical services provided outside of hospitals by both psychiatrists and general practitioners are not covered by private health insurance funds as Australia's public insurance program, Medicare, pays a benefit. Capacity and resource constraints have been noted as barriers to accessing appropriate care, both in Australia and internationally (Australian Medical Association, 2018; Saxena et al 2007). In particular, the limited capacity of public hospital-based mental health services in Australia has resulted in a growth in expenditure on private mental health services with private health insurance fund expenditure on mental health services increasing by an average of $9.0 \%$ per year between 2010-11 and 2014-15 compared to 3.6\% for the Australian government over the same period, albeit from a smaller funding base (Australian Institute of Health and Welfare, 2017a).

There is little information on individuals' utilisation of privately funded mental health services. Despite the fact that performance indicators such as average length of admissions, follow-up care and readmission rates are published for public mental health services (Australian Institute of Health and Welfare, 2017b), the same indicators are not published for private services, and publicly available information on private mental health services is limited to utilisation statistics reporting the volume of activity. There are also administrative coding differences with same-day visits 
making up the majority of private hospital activity (77\%) compared to $13 \%$ of activity for public hospitals (Australian Institute of Health and Welfare, 2016). These sameday visits cover a range of services including individual consultations and group programs that may be provided, and classified, as outpatient or ambulatory services in the public hospital sector and in other countries (Australian Institute of Health and Welfare, 2018).

Achieving more integrated planning and delivery of mental health care is a national priority but currently, relatively little is known about mental health services delivered in the Australian private hospital sector (Australian Government Department of Health 2017). Significant work has been done on standardising the collection and reporting of information on mental health care provided in Australia's public sector through the development of the Australian Mental Health Care Classification (Independent Hospital Pricing Authority, 2018) and the Key Performance Indicators for Australian Public Mental Health Services (Australian Institute for Health and Welfare, 2017b). The private sector is lagging behind, despite growing activity in the sector. Mental health services delivered in the private sector need to be better understood, particularly hospital-based services, as private expenditure has increased steadily in recent years and private health insurance sector reforms introduced in 2017 by the Australian Government may exacerbate the incentive for consumers to access hospital-based services. Regulation changes will allow people to upgrade their insurance policies for mental health treatment in hospitals without waiting periods, but there have been no changes to improve access to community-based mental health care (Rosenberg, 2017). 
Private health insurance claims are a potential data source to provide information on private, hospital-based mental health service use. Under the Private Health Insurance Act (2007), hospital insurance policies must include a minimum benefit for mental health care. Private health insurance funds contribute to funding $88 \%$ of private hospital activity for mental health conditions (Australian Institute of Health and Welfare, 2017c). Insurance claims data can also be used to analyse utilisation patterns over time at the patient-level, information that has not previously been reported for patients accessing mental health care in private hospitals.

Focusing on the organisation of health service delivery and insurance status as factors affecting health service utilisation, this study describes the utilisation of privately funded mental health services. Utilisation patterns are presented for hospital-based services, examining the demographic characteristics of patients accessing services and the average levels of utilisation per patients in terms of number of overnight admissions and same-day visits, length of stay per admission and insurer costs. Following analysis, the implications of the profile findings and the appropriateness of using insurance claims data for analysing mental health service use, not only for research, but also for policy and planning decisions are considered. This study compares:

\section{Demographic and hospitalisation characteristics of patients with mental} health-related hospital claims: The characteristics of patients with a mental health-related hospital claim are compared to insured patients with other types of hospital claims.

2. Differences in service utilisation patterns for patients with mental healthrelated hospital claims: Trends in hospital claims for the group of patients with mental health-related hospital claims are examined in relation to: 
a) Mental health-related and non mental health-related hospital claims,

b) Combinations of care for mental health-related hospitalisations, and

c) Mental health claims in the 28 days following an overnight admission discharge. This time period was selected because follow-up indicators for public mental health services report follow-up within seven days and readmissions within 28 days (Australian Institute of Health and Welfare, 2017b).

\section{Methods}

\section{Data sources and study population}

The primary data source analysed was de-identified hospital claims data from a group of 13 Australian private health insurance funds. Claims data related to patients residing in all Australian states and territories for services provided between 1 May 2014 and 30 April 2016 in more than 60 private hospitals. The claims dataset analysed are primarily comprised of information provided by hospitals to facilitate the payment of insurance claims such as admission date, length of stay and clinical services provided. Diagnostic and clinical information are generated by hospital clinical coders based on medical records. Insurer records may be supplemented by medical practitioner, allied health professionals and patients who provide details on services provided and billing information. Each person and hospital admission had a unique identifier.

Data were reviewed for anomalies such as records with negative benefits paid and for duplicate records. Following consultation with insurer data managers, any records without a hospital admission identifier were classified as a claims benefit adjustment and were excluded from the analysis, as these records match the service dates of 
another record but have a negative benefit paid to adjust a previous payment. These processes resulted in the removal of 5,963 people (2.5\% of the study population). The final dataset analysed included 236,910 patients with at least one hospitalisation (574,589 hospitalisations in total).

In this study, a "patient” is defined as a person accessing privately funded mental health services from a hospital during the study period. Privately funded services are those for which private health insurance funds pay a benefit. Overnight admissions and same-day visits are used to distinguish between the two main types of hospital services provided based on length of stay. When referring to all hospital services, both overnight admissions and same-day visits, the term "mental health hospitalisation" is used. Only private hospital utilisation was included to examine mental health service utilisation (2b) due to differences in coding same-day visits in private and public hospitals. As shown in Figure 1, the majority of patients (97.8\% or 3,137 patients) accessed mental health services in a private hospital. Psychology claims were included in the analysis examining mental heath-related claims following discharge from an overnight admission (2c).

\section{Data analysis}

Insurance claims were analysed using both cross-sectional and longitudinal analysis methods. Data analysis occurred in multiple stages described below. All statistical analysis was conducted using R version 3.2.2 (The R Foundation, 2018). 


\section{Demographic and hospitalisation characteristics of patients with mental health-}

related hospital claims

Patient classification codes were used as the primary method to categorise the group of patients with a mental health-related hospitalisation. Claims records coded as "PY" denote a mental health-related (or psychiatric) hospitalisation. Although mental health diagnosis information recorded as Australian-Refined Diagnosis Related Group codes (AR-DRG) was included for some claims records (Australian Consortium for Classification Development, 2018), the information was not consistently available for all claims records so the analysis could not examine differences in utilisation patterns based on the type of mental health condition. AR-DRG information, where available, was used to check for missing mental health-related hospitalisations. Two additional hospitalisations were found by searching codes relating to mental health or drug and

alcohol use. The list of specific diagnostic codes used is included as an Appendix. All patients with at least one overnight admission or same-day visit not categorised as mental health-related were included in the comparison group of patients without a mental health-related hospitalisation.

The demographic and hospital resource utilisation characteristics of the two groups were compared. The demographic variables analysed were sex, age and location of residence. Hospital resource utilisation was measured by the following indicators: number of same-day visits and overnight admissions per patient, average length of stay per admission and hospital costs paid by the insurer per patient. Same-day visits, where hospital admission and separation dates occur on the same day, were allocated a length of stay of one day consistent with methods for calculating national hospital statistics (Australian Institute of Health and Welfare, 2016). A measure of bed days was calculated by summing the length of stay for overnight admissions for each 
patient. Annual measures for resource utilisation were obtained by halving total results for the two-year study period. Pearson Chi-square $\left(\chi^{2}\right)$ analyses were used to compare categorical variables and Wilcoxon rank-sum (Mann-Whitney) tests were used for continuous variables, as variables were not normally distributed.

2. Differences in service use for patients with mental health-related hospital claims 2a) Mental health-related and non-mental health-related claims Hospital use for the group with at least one mental health-related hospital claim was analysed. Two groups were formed based on whether an individual had any nonmental health-related hospitalisations. Demographic and hospitalisation characteristics were compared using the same statistical methods described above in section 1.

\section{2b) Combinations of care for mental health-related hospitalisations}

Hospital use for the mental health group was further analysed with three groups formed according to whether a person claimed for only same-day visits, only overnight admissions or a combination of same-day visits and overnight hospital admissions in the study period. For the two groups with overnight admissions, hospital utilisation characteristics were compared using the same statistical methods

described above in section 1 . For the group with overnight admissions and same-day visits, analysis was completed on the proportion of patients with their first hospitalisation as an overnight admission followed by a same-day visit and vice versa.

\section{2c) Mental health-related claims following an overnight hospital admissions}

To investigate claims following an overnight hospital admission, we investigated each patient's first claim in the study period, recognising that the dataset covered a two- 
year period and patients may have multiple hospital claims during the study period. For patients with an overnight hospitalisation as their first hospitalisation in the dataset $(n=2,101,67 \%$ of patients with a private mental health-related hospitalisation), we analysed the type and timing of the next mental health-related claim, specifically whether the claim occurred within 28 days after discharge. Patients that were discharged from their first overnight admission within 28 days of the end of the study period were excluded from analysis. Overnight admissions, same-day visits and community-based psychology services claimed on private health insurance general treatment policies (for a limited range of non-hospital services) were included in the analysis.

\section{Ethics}

Ethics approval for this study was obtained from the University Human Research Ethics Committee.

\section{Results}

1. Demographic and hospitalisation characteristics of patients with mental healthrelated hospital claims

Table 1 compares demographic and hospital resource utilisation statistics for the group of patients with a mental health-related hospital claim $(n=3,209)$ and the comparison group with other types of hospital claims $(n=233,701)$. 
Table 1. Descriptive statistics for patients with and without mental health-related hospitalisations

1a) Demographic characteristics

\begin{tabular}{|c|c|c|c|}
\hline & $\begin{array}{l}\text { Mental health } \\
\text { hospitalisation } \\
(n=3,209)\end{array}$ & $\begin{array}{l}\text { No mental health } \\
\text { hospitalisation } \\
(n=233,701)\end{array}$ & $p$-value \\
\hline \multicolumn{4}{|l|}{ Sex $^{\mathrm{a}}$} \\
\hline Female, n (\%) & $1,990(62.0)$ & $130,432(55.8)$ & $<0.01$ \\
\hline \multicolumn{4}{|l|}{ Age groups $^{\mathrm{a}}$} \\
\hline $0-24, \mathrm{n}(\%)$ & $380(11.8)$ & 29,925 (12.8) & $<0.01$ \\
\hline $25-34, \mathrm{n}(\%)$ & $627(19.5)$ & $20,269(8.7)$ & \\
\hline $35-44, \mathrm{n}(\%)$ & $587(18.3)$ & $24,015(10.3)$ & \\
\hline $45-54, \mathrm{n}(\%)$ & $568(17.7)$ & 25,935 (11.1) & \\
\hline $55-64$, n (\%) & $559(17.4)$ & $47,501(20.3)$ & \\
\hline 65-74, n (\%) & $343(10.7)$ & $50,959(21.8)$ & \\
\hline $75+, \mathrm{n}(\%)$ & $145(4.5)$ & $35,097(15.0)$ & \\
\hline \multicolumn{4}{|l|}{ Location of residence $^{a}$} \\
\hline New South Wales, n (\%) & $1,343(41.9)$ & $85,564(36.6)$ & $<0.01$ \\
\hline Victoria, n (\%) & $1,024(31.9)$ & $70,500(30.2)$ & \\
\hline Queensland, n (\%) & $592(18.4)$ & $45,228(19.4)$ & \\
\hline $\begin{array}{l}\text { All other states and territories, } n \\
(\%)\end{array}$ & $250(7.8)$ & 32,409 (13.9) & \\
\hline
\end{tabular}

1b) Hospital resource utilisation characteristics

\begin{tabular}{|c|c|c|c|}
\hline & $\begin{array}{l}\text { Mental health } \\
\text { hospitalisation } \\
(n=3,209)\end{array}$ & $\begin{array}{l}\text { No mental health } \\
\text { hospitalisation } \\
(n=233,701)\end{array}$ & $p$-value \\
\hline Total hospitalisations (two years), $\mathrm{n}$ & 33,134 & 541,455 & \\
\hline $\begin{array}{l}\text { Overnight admissions }{ }^{\mathrm{a}} \text {, n (\% } \\
\text { total) }\end{array}$ & $7,321(22.1)$ & $181,516(33.5)$ & $<0.01$ \\
\hline Same-day visits, n (\% total) & $25,813(77.9)$ & $359,939(66.5)$ & \\
\hline $\begin{array}{l}\text { Mean annual overnight admissions } \\
\text { per patient }{ }^{\text {b,c }}, \mathrm{n}(\mathrm{SD})\end{array}$ & $1.3(1.2)$ & $0.8(0.6)$ & $<0.01$ \\
\hline $\begin{array}{l}\text { Mean annual same-day visits per } \\
\text { patient }^{\mathrm{b}, \mathrm{d}}, \mathrm{n}(\mathrm{SD})\end{array}$ & $6.4(8.9)$ & $1.1(3.6)$ & $<0.01$ \\
\hline $\begin{array}{l}\text { Mean length of stay for overnight } \\
\text { admissions }{ }^{\text {b }} \text {, days (SD) }\end{array}$ & $15.0(14.1)$ & $4.6(7.3)$ & $<0.01$ \\
\hline $\begin{array}{l}\text { Mean annual bed days for overnight } \\
\text { admissions per person b,c , days (SD) }\end{array}$ & $19.7(20.3)$ & $3.6(7.2)$ & $<0.01$ \\
\hline $\begin{array}{l}\text { Mean annual hospital costs paid by } \\
\text { insurer }^{\mathrm{b}}, \mathrm{A} \$(\mathrm{SD})\end{array}$ & $\begin{array}{l}\$ 13,192 \\
(13,457)\end{array}$ & $\$ 2,065(4,346)$ & $<0.01$ \\
\hline \multicolumn{4}{|c|}{$\begin{array}{l}\text { Abbreviations: SD: standard deviation } \\
\text { a Pearson Chi-square }(\chi 2) \text { test used to compare categorical and proportional variables. } \\
\text { b Wilcoxon rank-sum (Mann-Whitney) test used to compare means of continuous }\end{array}$} \\
\hline
\end{tabular}


${ }^{\mathrm{c}}$ Mean calculated based on patients with an overnight admission (2,795 patients with mental health-related admissions and 115,910 patients with other admissions)

${ }^{\mathrm{d}}$ Mean calculated based on patients with a same-day visit (2,021 patients with mental health-related visits and 162,907 patients with other visits).

The group with mental health-related hospital utilisation has a greater proportion of females and are also significantly younger. Only 32.6\% of the group with mental health-related hospital utilisation are aged 55 years and over compared to $57.1 \%$ of the group with other types of hospitalisations. Geographically, there are relatively more patients with a mental health-related hospital claim in New South Wales compared to other types of hospital claims, and relatively fewer patients from states and territories other than New South Wales, Victoria and Queensland with a mental health-related hospital claim compared to other types of hospital claims.

The mental health group has a significantly higher level of hospital use for all utilisation measures. On average, the annual number of bed days for overnight admissions for the mental health group is 19.7 days compared to 3.6 days for the group with other types of hospitalisations. The mental health group also has an annual average of 6.4 same-day visits compared to 1.1 for the group with other types of hospitalisations. Mean annual hospital costs paid by the insurer are six times higher for the mental health group. The standard deviation for all utilisation statistics is greater for the mental health group, indicating a higher level of variation in hospital utilisation within the mental health group. 


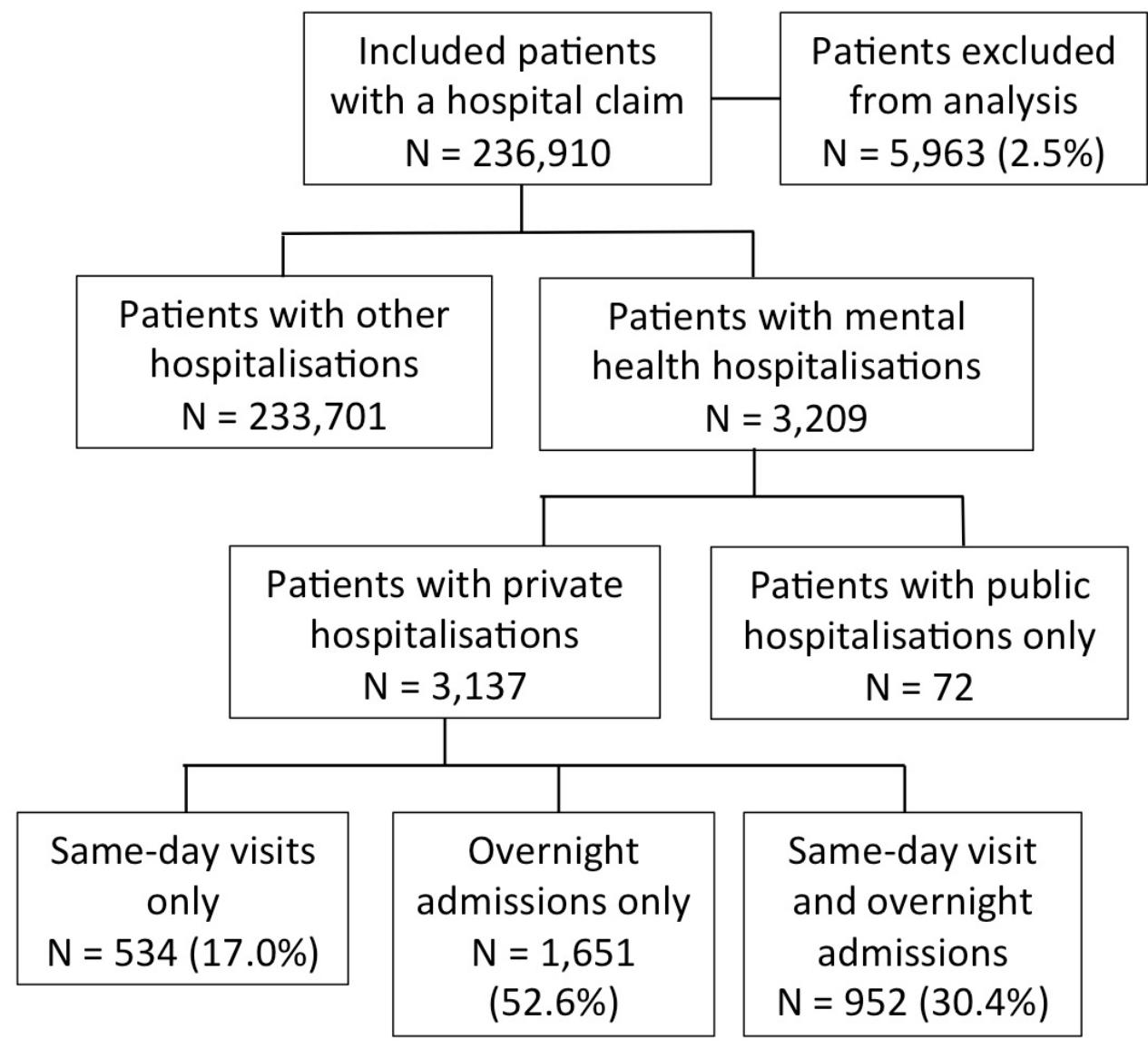

Figure 1. Study population with hospitalisations for 2014-2016 showing patients with mental health-related hospitalisations

2. Differences in service use for patients with mental health-related hospital claims

2a) Mental health-related and non-mental health-related claims

Table 2 compares demographic and hospital resource utilisation characteristics within the mental health group for two groups - patients with only mental health-related hospitalisations and patients with mental health-related and other hospitalisations. Just over half of the mental health group (1,633 patients, 51\%) only claim for mental health-related hospitalisations. The group with only mental health-related hospitalisations are significantly younger and less likely to be female. Although the group with only mental health-related hospitalisations has a significantly longer length of stay per overnight admission and higher average annual number of sameday visits, the group with mental health-related and other hospitalisations has a 
significantly higher average number of overnight admissions per year, higher average number of bed days per year for overnight admissions and significantly higher average hospital costs paid by insurers per year. Overall, 64\% hospitalisations for the group with both mental health-related and other types of hospitalisations are mental health-related.

Table 2. Descriptive statistics for patients with mental health-related hospitalisations, with and without other types of hospitalisations

\begin{tabular}{|c|c|c|c|}
\hline & $\begin{array}{l}\text { Only mental health } \\
\text { hospitalisations } \\
(n=1,633)\end{array}$ & $\begin{array}{l}\text { Mental health and } \\
\text { other } \\
\text { hospitalisations } \\
(n=1,576)\end{array}$ & $p$-value \\
\hline Female $^{\mathrm{a}}, \mathrm{n}(\%)$ & $963(59)$ & $1,024(65)$ & $<0.01$ \\
\hline Mean age $^{\text {b }}$, years (SD) & $42.2(15.6)$ & $49.1(17.2)$ & $<0.01$ \\
\hline $\begin{array}{l}\text { Total hospitalisations (two } \\
\text { years), } n\end{array}$ & 12,473 & 20,661 & \\
\hline $\begin{array}{l}\text { Overnight admissions }{ }^{\mathrm{a}}, \mathrm{n} \\
\text { (\% total) }\end{array}$ & $2,288(18)$ & $5,031(24)$ & $<0.01$ \\
\hline $\begin{array}{l}\text { Same-day visits, n (\% } \\
\text { total) }\end{array}$ & $10,185(82)$ & $15,630(76)$ & \\
\hline $\begin{array}{l}\text { Mean annual overnight } \\
\text { admissions per patient }{ }^{\mathrm{b}, \mathrm{c}}, \mathrm{n} \\
\text { (SD) }\end{array}$ & $0.9(0.8)$ & $1.7(1.4)$ & $<0.01$ \\
\hline $\begin{array}{l}\text { Mean annual same-day } \\
\text { visits per patient, }{ }^{\mathrm{b}, \mathrm{d}} \text {, n (SD) }\end{array}$ & $6.6(7.7)$ & $6.3(9.6)$ & $<0.01$ \\
\hline $\begin{array}{l}\text { Mean length of stay for } \\
\text { overnight admissions }{ }^{\mathrm{b}} \text {, } \\
\text { days (SD) }\end{array}$ & $18.7(13.5)$ & $13.3(14.1)$ & $<0.01$ \\
\hline $\begin{array}{l}\text { Mean annual bed days for } \\
\text { overnight admissions per } \\
\text { patient }^{\mathrm{b}, \mathrm{c}} \text {, days (SD) }\end{array}$ & $16.2(15.5)$ & $22.8(23.3)$ & $<0.01$ \\
\hline $\begin{array}{l}\text { Mean annual hospital } \\
\text { costs paid by insurer }{ }^{\mathrm{b}}, \$ \\
\text { (SD) }\end{array}$ & $\$ 10,071(10,599)$ & $\$ 16,425(15,228)$ & $<0.01$ \\
\hline \multicolumn{4}{|c|}{$\begin{array}{l}\text { Abbreviations: SD: standard deviation } \\
\text { a Pearson Chi-square ( } \chi 2) \text { test used to compare categorical and proportional variables } \\
\text { b Wilcoxon rank-sum (Mann-Whitney) test used to compare means of continuous } \\
\text { variables } \\
{ }^{c} \text { Mean calculated based on patients with an overnight admission ( } 774 \text { patients with } \\
\text { only mental health-related hospitalisations and } 1,248 \text { patients with mental health- } \\
\text { related and other hospitalisations) } \\
\text { d Mean calculated based on patients with a same-day visit (1,322 patients with only } \\
\text { mental health-related hospitalisations and 1,472 patients with mental health-related } \\
\text { and other hospitalisations). }\end{array}$} \\
\hline
\end{tabular}




\section{b) Combinations of care for mental health-related hospitalisations}

Categorising patients admitted to private hospitals according to the type of mental health-related hospitalisation claimed during the study period shows the majority of patients (52.6\%) only claim for overnight admissions, 30.4\% of patients claim for both same-day visits and overnight admissions and $17.0 \%$ of patients only claim for same-day visits. The number of patients for the three groups is shown in Figure 1. Eighty percent of all hospitalisations are same-day visits. For the two groups with overnight admissions in the study period, the group of patients with both overnight admissions and same-day visits have significantly higher levels of service use with an average of 1.2 admissions per year $(\mathrm{SD}=1.1)$ and an average of 20.1 days per overnight admission $(\mathrm{SD}=14.1)$, compared to 0.9 admissions per year $(\mathrm{SD}=0.9$, $p<0.01)$ and 17.8 days per overnight admission ( $\mathrm{SD}=13.9, p<0.01)$ for the overnightonly group. For the 952 patients with both overnight admissions and same-day visits, 78\% have an overnight admission occur first in the study period followed by a sameday visits and 22\% have a same-day visit first followed by an overnight admission.

\section{c) Mental health claims following an overnight hospital discharge}

Of the 2,101 patients with an overnight admission as their first hospitalisation during the study period, Figure 2 shows the majority of patients (68\%) do not claim for additional mental health-related services on their private health insurance in the 28 days following discharge. Nineteen percent of patients claim for a same-day visit within 28 days and 12\% of patients claim for another overnight admission. Only 1\% of patients claim for a psychology consultation as their first claim after hospital discharge within 28 days. 


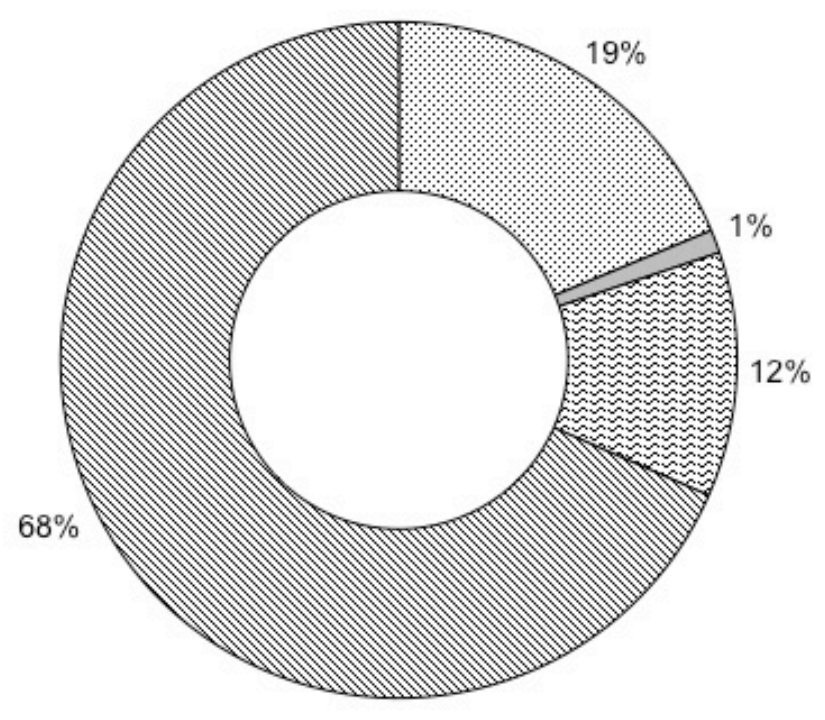

Same-day

口Psychology

图Overnight

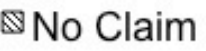

Figure 2. Service claims within 28 days after discharge from first overnight hospital admission

\section{Discussion}

In this sample of privately insured patients, the group of patients with mental healthrelated hospital claims only account for $1.4 \%$ of all patients with a hospitalisation. The group with mental health-related hospital claims are significantly younger and have significantly higher levels of hospital utilisation compared to the group of patients with claims for other types of hospitalisations. Access to, and use of, appropriate care should be encouraged for this group. The type of mental healthrelated hospital claims varies across the insured population. Rather than most patients accessing both overnight admissions and same-day visits as would be expected if same-day visits were primarily provided as follow-up care in conjunction with treatment provided during overnight admissions, this study finds three distinct groups of service users - same-day visits only, overnight admissions only and a combination of same-day visits and overnight admissions. More than half of the patients with a mental health-related hospitalisation only claim for overnight admissions in the two- 
year study period and only one third of patients make an additional mental healthrelated private health insurance claim within 28 days after discharge from their first overnight admission in the study period.

The study findings report measures of utilisation including the type, combination and frequency of mental health services obtained by patients with private health insurance. It is more challenging to understand the specific factors associated with the utilisation patterns observed and the outcomes of the care received as discussed further in the next section, which focuses on the implications for research, and policy considerations for the private health insurance sector.

Interpretation of mental health service utilisation patterns using insurance claims data is limited

The study findings reveals new information on the demographic characteristics of patients accessing private mental health services and patterns of mental health service use in private hospitals in Australia. The organisation of mental health service delivery in the private sector and insurance regulation seem to affect utilisation of services but the specific influence of clinical, organisation and policy factors that may affect the use of mental health services cannot be ascertained due to limitations in the data collected by private health insurers. For example, the mix of claims for overnight and same-day hospitalisations, specifically the high proportion of patients with only overnight admission claims, may be affected by a number of different factors as outlined in the Andersen Model - patient need, insurance policy coverage, hospital treatment policies or service availability. All of these factors may be important contributors to mental health service use but the influence of these different factors on service utilisation cannot be distinguished using the claims data analysed. National 
policy factors may also contribute to patterns of private mental health service use. The current Australian regulatory environment may influence service use as insurers can only pay benefits for mental health-related medical services provided in hospitals but not in the community (although services of other health professionals provided in the community such as psychologists may be paid by insurers).

The fragmented funding of mental health care is reflected in the type of services claimed on private health insurance

The profile reveals only a limited picture of the care journey of people with mental health conditions. The profile limitations also reflect the complex financing mechanisms that people with a mental health condition and their carers have to navigate to access different forms of mental health care. The finding that only one third of patients made a mental health-related claim within 28 days after discharge cannot be interpreted that patients are not receiving any mental health services, just that they are not claimed on private health insurance. However, the extent of Medicare-funded mental health care that privately insured people access is not known. Ideally, patients would be connected with community-based support as part of hospital discharge regardless of funding arrangements, consistent with stepped care models (Cross and Hickie, 2017), but the extent of this care coordination in practice cannot be assessed using private health insurance claims data alone.

A more comprehensive picture of mental health treatment in the private sector could be built though analysing linked private health insurance and Medicare Benefits Schedule data. This is an area for future research that would capture communitybased psychiatry services and mental health services funded through the Better Access scheme that funds a limited amount of general practitioner-referred 
psychological care (Australian Government Department of Health, 2012). Although partially funded through government sources, health professionals operating in private practice mainly provide these services. Initiatives to develop and analyse a comprehensive record of service utilisation using multiple, linked data sources are also relevant for researchers in other jurisdictions with a mix of public and privately funded health services.

Claims data provides little information on the outcomes of mental health care Monitoring care following hospital discharge is difficult using claims data. There are no indicators of other outcomes of mental health care in claims data, despite the fact measures are collected for the private sector via the Australian Private Hospitals Association Private Psychiatric Hospitals Data Reporting and Analysis Service (PPHDRAS). The standardised measures of patients' clinical status collected include the Health of the Nation Outcome Scale (HoNOS) administered by clinicians and the Mental Health Questionnaire-14 completed by consumers (Burgess et al 2015).

However, reports on these outcome measures for the private sector are released to the public irregularly and participation by hospitals is voluntary (Morris-Yates, 2016). There is an opportunity to better understand outcomes of mental health care delivered in private hospitals through the longitudinal analysis of this data collection, although as for claims data, data quality needs to be assessed.

This study provides a description of patient-level utilisation patterns in private hospitals for mental health care but is limited in providing information on care continuity between hospital and community-based services and the factors influencing patterns of hospital use. There is a need for greater transparency and accountability of the care delivered in the private sector given the government funds $75 \%$ of the 
scheduled Medicare fee for medical services delivered in private hospitals (Australian Government Private Health Insurance Ombudsman, 2018), and contributes more than \$6 billion annually via the private health insurance premiums rebate (Australian Government Department of Health, 2016).

Based on analyses using insurance claims data, we find that private health insurance funds have little visibility of the mental health care that patients receive post hospital discharge. It is encouraging to observe that a number of private health insurers have started to introduce mental health support programs for patients leaving hospital such as the MindStep program (Remedy Healthcare, 2018). However, determining participant eligibility, assessing individual care needs and ensuring alignment with a patient's other mental health care providers are important considerations for insurers offering these programs. Additionally, these mental health support programs are generally short-term (lasting only several weeks) so programs need to be evaluated to assess their impact on health outcomes and service use.

\section{Limitations}

This study has a number of limitations. This study used two years of insurance claims data (2014-2016) but does not account for service utilisation before or after that period. This is a practical limitation of using real world, insurance claims data for research. While such routinely collected data are appealing data sources for research because of their large size, their longitudinal perspective, and their event-based information (Smeets et al., 2011), inadequate attention is often given to the quality of data sources, such as claims data, and the challenges to reliably use the data (Benchimol et al., 2013). 
Research examining longitudinal service utilisation accessed by patients over a longer time period and the factors influencing access to care would be a valuable area of future research. Additionally, clinical diagnoses were not consistently recorded in the claims dataset analysed so differences in patterns of service utilisation of patients with different types of mental health conditions could not be analysed. The sample of private health insurance funds in this study represents approximately $10 \%$ of the insured population in Australia, therefore how representative the study findings are of the broader insured population needs to be considered. The insurance claims sample used in this study is comparable to the national private health insurance market, with regard to member age (69\% hospital admissions for people aged 55 years and over versus 64\% nationally), sex (females account for 55\% of admissions versus 54\% nationally) and location of residence (36\% hospital admissions occur in New South Wales versus 32\% nationally) (Australian Prudential Regulation Authority, 2017).

\section{Conclusion}

Utilisation patterns and performance information on mental health services delivered by the Australian private hospital sector is lacking, despite increasing activity over the last decade. Examining insurance claims data from a sample of private health insurance funds shows that although the proportion of the insured population claiming for mental health-related hospital services is small, hospital resource utilisation for this patient group is much higher than for patients with claims for other types of hospital admissions. Examining the potential factors contributing to patterns of mental health-related hospital claims, such as the large proportion of patients that only claim for overnight admission, is challenging due to the scope of claims data and the regulated role of the Australian private health insurance sector in paying benefits for health care. The factors contributing to trends in mental health service utilisation in 
private hospitals, and the performance and outcomes of care, needs to be better understood given the government is a significant funder of these services. Linking multiple data sources on service use and outcomes is required as insurance claims data only provide information on a limited part of service utilisation. 


\section{References}

Aday, L.A. and Andersen, R.M. (1974), “A Framework for the Study of Access to Medical Care”, Health Services Research, Vol. 9, No. 3, pp. 208-20.

Andersen, R.M. (1995), "Revisiting the Behavioral Model and Access to Medical Care: Does it Matter?”, Journal of Health and Social Behavior, Vol. 36, pp. 110.

Andersen, R.M., Davidson, P.M. and Baumeister, S.E. (2014), "Improving access to care”, in Kominski, G.F. (Ed.), Changing the U.S. health care system: Key issues in health services policy and management, Jossey-Bass, San Francisco, CA, pp 33-69.

Australian Consortium for Classification Development (2018), “Australian Refined Diagnosis Groups version 9.0", available at: https://www.accd.net.au/ArDrg.aspx?page=6 (accessed 13 February 2018).

Australian Government Department of Health (2012). "Better access to mental health care: fact sheet for patients", available at:

http://www.health.gov.au/internet/main/publishing.nsf/Content/mental-bafact-pat (accessed 19 June 2017).

Department of Health and Ageing (2013) "National Mental Health Report 2013: tracking progress of mental health reform in Australia 1993 - 2011”, Canberra, Australia.

Australian Government Department of Health (2016). "Private health insurance and prostheses committees", available at: http://www.health.gov.au/internet/budget/publishing.nsf/Content/budget2016factsheet14.htm (accessed 30 January 2018).

Australian Government Department of Health (2017). The Fifth National Mental Health and Suicide Prevention Plan. Canberra, Australia.

Australian Government Private Health Insurance Ombudsman (2018), "PrivateHealth.gov.au: What is covered?", available at: https://www.privatehealth.gov.au/healthinsurance/whatiscovered/ (accessed 13 February 2018).

Australian Institute of Health and Welfare (2016), “Admitted patient care 2014-15: Australian hospital statistics”, Health services series no. 68. Cat. no. HSE 172. Canberra: AIHW.

Australian Institute of Health and Welfare (2017a), "Mental health services in Australia: Expenditure on mental health services”, available at: https://mhsa.aihw.gov.au/resources/expenditure/specialised-mh-services/ (accessed 9 June 2017).

Australian Institute of Health and Welfare (2017b), "Key Performance Indicators for Australian Public Mental Health Services”, available at: https://www.aihw.gov.au/reports/mental-health-services/mental-healthservices-in-australia/mental-health-indicators/key-performance-indicators-foraustralian-public-mental-health-services (accessed 29 January 2018).

Australian Institute of Health and Welfare (2017c), "Private health insurance use in Australian hospitals, 2006-07 to 2015-16: Australian hospital statistics”, Health Services Series no. 81. Cat. no. HSE 196. Canberra: AIHW.

Australian Institute of Health and Welfare (2018), "Key concepts: Same day admitted mental health care”, available at: https://www.aihw.gov.au/reports/mentalhealth-services/mental-health-services-in-australia/report-contents/same-dayadmitted-mental-health-related-care/key-concepts\#20_same_day (accessed 6 June 2018). 
Australian Medical Association (2018), “Position Statement: Mental Health 2018”, available at: https://ama.com.au/position-statement/mental-health-2018 (accessed 6 June 2018).

Australian Prudential Regulation Authority (2017), "Private Health Insurance Statistical Trends March 2017 - Benefit”, available at: http://www.apra.gov.au/PHI/Publications/Pages/Statistical-Trends.aspx (accessed 9 June 2017).

Benchimol, E.I., Langan, S. and Guttmann, A. (2013), “Commentary: Call to RECORD: the need for complete reporting of research using routinely collected health data”, Journal Of Clinical Epidemiology, Vol. 66, pp. 703-5.

Burgess, P., Pirkis, J. and Coombs, T. (2015), "Routine outcome measurement in Australia”, International Review of Psychiatry, Vol. 27, No. 4, pp. 264-75.

Cross, S. and Hickie, I .(2017), “Transdiagnostic stepped care in mental health,” Public Health Research \& Practice, Vol. 27, No. 2, e2721712.

Independent Hospital Pricing Authority (2018), “Classifications: mental health care”, available at: https://www.ihpa.gov.au/what-we-do/mental-health-care (accessed 21 February 2018).

Morris-Yates, A. (2016), "Private hospital-based psychiatric services, 1 July 2014 to 30 June 2015. PMHA-CDMS Annual Statistical Report”, available at: https://catalogue.nla.gov.au/Record/7129347 (accessed 30 January 2018).

Remedy Healthcare (2018), “MindStep Mental Health Program”, available at: https://www.remedyhealthcare.com.au/mental-health/ (accessed 30 January 2018).

Rosenberg, S. (2017), "Insurance changes not enough to drive real mental health reform”, The Conversation, 17 October, available at: https://theconversation.com/insurance-changes-not-enough-to-drive-realmental-health-reform-85753 (accessed 30 January 2018).

Saxena, S., Thornicroft, G., Knapp, M. and Whiteford, H. (2007), "Resources for mental health: scarcity, inequity, and inefficiency”, Lancet, Vol. 370, No. 9590, pp. 878-89.

Sharfstein, S. (1995), "The role of private insurance in financing treatment for depression", Social Psychiatry and Psychiatric Epidemiology, Vol. 30, No. 5, pp. 236-9.

Smeets, H.M., de Wit, N.J. and Hoes, A.W. (2011), "Routine health insurance data for scientific research: potential and limitations of the Agis Health Database", Journal Of Clinical Epidemiology, Vol. 64, No. 4, pp. 424-30.

The R Foundation 2017. The R Project for Statistical Computing, available at https://www.r-project.org/ (accessed 21 February 2018).

Vladu, C., Novac, A., Preda, A. and Bota, R. (2016), "No Health Without Mental Health”, Mental Illness, Vol. 8, No. 2, pp. 55-56. 
Appendix: Australian-Refined Diagnosis Related Codes (AR-DRG) for mental health disorders

U40 Mental Health Treatment W ECT, Sameday

U60 Mental Health Treatment W/O ECT, Sameday

U61 Schizophrenia Disorders

U62 Paranoia and Acute Psychotic Disorders

U63 Major Affective Disorders

U64 Other Affective and Somatoform Disorders

U65 Anxiety Disorders

U66 Eating and Obsessive-Compulsive Disorders

U67 Personality Disorders and Acute Reactions

U68 Childhood Mental Disorders

V60 Alcohol Intoxication and Withdrawal

V61 Drug Intoxication and Withdrawal

V62 Alcohol Use and Dependence

V63 Opioid Use and Dependence

V64 Other Drug Use and Dependence 